\title{
Review Article \\ Constraints on a Fourth Generation of Fermions from Higgs Boson Searches
}

\author{
Alexander Lenz ${ }^{1,2}$ \\ ${ }^{1}$ TH Division, Physics Department, CERN, CH-1211, Geneva 23, Switzerland \\ ${ }^{2}$ Institute for Particle Physics Phenomenology, Department of Physics, Durham University, Durham DH1 3LE, UK
}

Correspondence should be addressed to Alexander Lenz; alenz@cern.ch

Received 30 July 2012; Accepted 7 September 2012

Academic Editor: Johan Alwall

Copyright (C) 2013 Alexander Lenz. This is an open access article distributed under the Creative Commons Attribution License, which permits unrestricted use, distribution, and reproduction in any medium, provided the original work is properly cited.

\begin{abstract}
We review the past and current status of the extension of the standard model (SM) by a fourth generation of fermions. In particular the new results for Higgs boson searches at the LHC and at Tevatron exclude the possibility of having simply a perturbative fourth generation of fermions with one Higgs doublet (SM4). We also briefly mention more complicated extensions of the SM4, which are not yet excluded, like adding in addition another Higgs doublet to the SM4.
\end{abstract}

\section{Introduction}

In the standard model $[1,2]$ the masses of the $\mathrm{W}$ and $\mathrm{Z}$ bosons are generated via the so-called Higgs mechanism [3-7]. Also all fermion masses can be created by this mechanism via the Yukawa interaction. Schematically the Lagrangian of the SM reads

$$
\begin{aligned}
\mathscr{L}= & -\frac{1}{4} F_{\mu \nu} F^{\mu \nu}+i \bar{\Psi} \not \supset \Psi+\left|D_{\mu} \Phi\right|^{2} \\
& -V(\Phi)+i \bar{\Psi}_{i} Y_{i j} \Phi \Psi_{j}+\text { h.c. }
\end{aligned}
$$

The first term of (1) describes the gauge fields of the strong, weak, and electromagnetic interaction, the second term describes the massless fermions and their interaction with the gauge fields. The third and fourth term represent the free scalar field, the Higgs potential, and the interaction of the scalar field with the gauge fields. The special form of the Higgs potential will result in masses for some of the gauge bosons. The last term describes the interaction between fermions and the scalar field, the so-called Yukawa interaction. In the above equation the number of fermion generations is a free parameter. Experimentally three generations have been observed, but a priori there is no reason why there could not be more generations. The Yukawa interaction results in masses for all the fermions and also in the CabibboKobayashi-Maskawa (CKM) matrix [8,9], which describes the coupling of the quarks to the charged electroweak gauge bosons $W^{+}$and $W^{-}$. In the case of two fermion generations the CKM matrix consists of one real parameter (the socalled Cabibbo angle), in the case of three generations of three real parameters and one complex phase and in the case of four generations there are six real mixing angles and three complex phases. A complex coupling in the theory will give rise to $\mathrm{CP}$-violation and the $\mathrm{CKM}$ sector is the only part of the SM, where CP-violation has been experimentally detected. CP-violation is in particular interesting because it is one of the three Sakharov criteria [10-12] that allow the dynamical creation of the observed baryon asymmetry in the universe. In that sense, having three generations of fermions is a minimal requirement in order to have CP-violation in the fundamental laws of nature. It turns out however, that the amount of CP-violation in the CKM matrix with three generations is not sufficient to explain the baryon asymmetry, while the amount of CP-violation with four generations seems to be sufficient [13]. This clearly seems to be a nice motivation to study an extension of the SM with additional fermion generations.

Besides giving masses to the gauge bosons of the weak interaction and the fermions the Higgs mechanism predicts also in its simplest form the existence of one massive neutral scalar particle, the Higgs boson. The search for the Higgs boson was one of the primary goals of the LHC, which has 


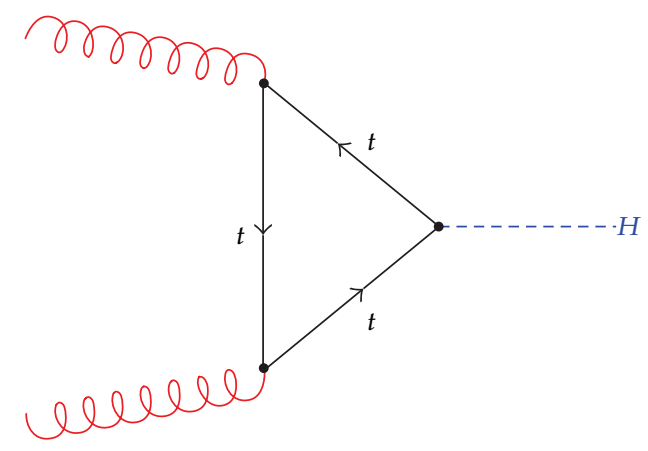

FIGURE 1: Gluon fusion with an internal top loop turns out to be the dominant Higgs production mechanism at the LHC.

probably been achieved in July 2012, when a new boson with a mass of about $125 \mathrm{GeV}$ was observed by ATLAS and CMS [14]. The properties of this new boson seem to be in accordance with the properties of SM Higgs boson, but to draw definite conclusions more data are needed.

At the LHC the dominant production mechanism of the Higgs boson is gluon fusion [19], where two gluons couple to a top triangle, which again couples to a Higgs boson, see Figure 1 (taken from [20]) - for a review of Higgs boson production and decay, see [21]. This process has been studied in detail in the standard model and many corrections to it have been determined. Leading QCD corrections have been calculated already in 1991 [22-24] and they turned out to be huge. Depending on the Higgs mass, they can be up to $100 \%$. Hence, it was necessary to calculate also NNLO QCD corrections [25-37]. Also NLO-electroweak and mixed QCD-electroweak corrections were determined for the gluon fusion [38-44].

At the LHC one is, however, also sensitive to subdominant production processes like associated vector boson-Higgs production/Higgs-Strahlung $(p p \rightarrow V H)$ [45, 46], vector boson fusion $(p p \rightarrow q q H)$ [47], and top-Higgs production $(p p \rightarrow t H)$.

End of 2011 some hints for the existence of a Higgs-like boson at a mass of about $125 \mathrm{GeV}$ have been presented by ATLAS [48-56] and CMS [57-65]. The strongest signal were found in the $H \rightarrow \gamma \gamma$ and $H \rightarrow Z Z^{*}$ channel, but also $H \rightarrow W W, H \rightarrow \tau^{+} \tau^{-}$and $H \rightarrow b \bar{b}$ was investigated. In March 2012 Tevatron presented also small hints for a Higgs boson via the reaction $p+\bar{p} \rightarrow V H \rightarrow V b \bar{b}$ in the same mass region [66]. On July 2, 2012 Tevatron updated their result and they found an even stronger indication for $H \rightarrow b \bar{b}$ [67]. Compared to March 2012 the statistical significance increased from 2.6 standard deviations to 2.9 standard deviations. Finally ATLAS and CMS announced on July 4, 2012 the observation of a new Higgs-like boson [14]. ATLAS presented results for the decay channels $H \rightarrow \gamma \gamma$ and $H \rightarrow Z Z^{*}$, (Recently ATLAS made also some data for the channel $H \rightarrow W W$ public in ATLAS-CONF-2012-098.) obtaining a statistical significance of 5.0 standard deviations for a boson with a mass of $126.5 \mathrm{GeV}$. CMS included in addition $H \rightarrow W W, H \rightarrow \tau^{+} \tau^{-}$, and $H \rightarrow b \bar{b}$, obtaining a statistical significance of 4.9 standard deviations for a boson with a mass of $125.3 \pm 0.6 \mathrm{GeV}$. The couplings agree within errors with the SM expectations, although the signal strength for the $H \rightarrow \gamma \gamma$ channel is enhanced in both ATLAS $(1.9 \pm 0.5)$ and CMS $(1.56 \pm 0.43)$. Here more data are needed to investigate whether this is a statistical fluctuation or a first glimpse of physics beyond the SM. These results have profound implications on the physics of a fourth generation of fermions, which will be discussed below.

\section{Higgs Production and Decay within the SM4}

2.1. The Model. We define the SM4 as the extension of the usual standard model simply by an additional chiral family of fermions. Its fermionic contents reads

$$
\left(\begin{array}{l}
u \\
d
\end{array}\right)\left(\begin{array}{l}
c \\
s
\end{array}\right)\left(\begin{array}{l}
t \\
b
\end{array}\right)\left(\begin{array}{l}
t^{\prime} \\
b^{\prime}
\end{array}\right) \quad\left(\begin{array}{c}
v_{e} \\
e^{-}
\end{array}\right)\left(\begin{array}{c}
v_{\mu} \\
\mu^{-}
\end{array}\right)\left(\begin{array}{l}
v_{\tau} \\
\tau^{-}
\end{array}\right)\left(\begin{array}{l}
v_{4} \\
l_{4}^{-}
\end{array}\right) .
$$

In addition one assumes Dirac masses for the heavy neutrinos, only one Higgs doublet, and the perturbativity of the Yukawa couplings. The strong conclusions we will make about the exclusion of the SM4 rely on all these assumptions, so it is important to keep them in mind. For some reviews of this model see, for example, [68, 69].

Extending the SM by an additional fermion generation might change many observables directly, but also indirectly via loop processes. Thus, the SM4 is subjected to a number of constraints:

(1) direct searches for the production of heavy quarks and leptons of the fourth generation at the LHC and at Tevatron;

(2) flavor observables are affected by a fourth generation via a change of the values of the CKM elements and via loop processes;

(3) electroweak precision observables are affected by a fourth generation via loop processes;

(4) higgs production and decay are affected by a fourth generation via loop processes.

The first three classes of constraints will be discussed briefly and the last class in detail below.

2.2. The Numerous Deaths of the SM4. The SM4 was already killed many times in the literature, however, until very recently the arguments for the exclusion of the SM4 relied always on some additional unjustified assumptions, which will be elaborated below. In the 1980s the idea of a fourth generation was relatively popular, see, for example, [70-81]. Later on this possibility was widely considered not to be attractive, see, for example, [82] for an early reference, where arguments like "Rather than being nice the presence of a 4th family is likely to be an hindrance for our understanding of the patterns in flavor physics.", were given against the existence of the fourth generation. The scientific content of such statements is clearly questionable.

After LEP measured the number of light neutrinos to be very close to three, $N_{v}=2.9840 \pm 0.0084$ [83], this was 
considered to be a further argument against the fourth generation, because it was implicitly assumed that the neutrino of the fourth generation is massless as well. The actual bound from LEPI was, however, only valid for light neutrinos, with $m_{v}<M_{Z} / 2$. After the observation that neutrinos have mass $\left(m_{v} \neq 0\right)$, see, for example, $[84,85]$, it was still considered to be unnatural that the fourth neutrino should be heavy $m_{v}>M_{Z} / 2$, while the first three ones are very light. We would like to stress here, however, that it is not excluded by any principle that nature itself is unnatural in the above sense!

Electroweak precision observables are also very sensitive to loop effects of the new heavy fermions. In the PDG article from Langacker and Erler [83] the effects of the SM4 to the Peskin and Takeuchi parameters $[86,87] S$ and $T$ were investigated. Concretely, these were the 1-loop contributions to the $W$ - and $Z$-boson and the $\gamma$ self-energies with virtual $t^{\prime}{ }^{\prime}$, $b^{\prime}$-quarks or virtual $v_{4}$ and $l_{4}$. For a long time the PDG article from Langacker and Erler (see, e.g., the archive of [83]) gave the wrong impression that a fourth generation is excluded by electroweak constraints. We list here some statements of previous PDG versions.

(i) 1994: one heavy generation of ordinary fermions is allowed at 95\% CL.

(ii) 1998: an extra generation of ordinary fermions is now excluded at the $99.2 \%$ CL.

(iii) 2002: an extra generation of ordinary fermions is excluded at the $99.8 \% \mathrm{CL}$ on the basis of the $S$ parameter alone [...]. This result assumes [...] that any new families are degenerate. This restriction can be relaxed [...] to $95 \%$.

(iv) 2010: An extra generation of SM fermions is excluded at the $6 \sigma$ level on the basis of the $S$ parameter alone $[\ldots]$. This result assumes that $[\ldots]$ any new families are degenerate [...]. Thus, a fourth family is disfavored, but not excluded by current data.

Concerning some of the above statements one should keep in mind that there is no reason at all to consider the $S$ parameter alone and there is also no reason to consider only a degenerate fourth family. Thus, we consider the statements, which were made under these assumptions, to be misleading.

So for quite some time it was an established prejudice; that the SM4 is excluded. This situation changed quite significantly with the paper of Kribs et al. from 2007 [88]. This paper marked somehow the resurrection of the SM4. Till July 2012 it was cited more than 300 times, showing the renewed interest in the physics of a fourth family. The authors showed in their paper that the SM4 is phenomenologically not excluded, in particular it is not in conflict with electroweak precision observables. In more details they noticed that a change of the Peskin and Takeuchi $[86,87]$ parameter $S$ can be compensated to a large extent by a similar change in the parameter $T$, that is, $\Delta S \approx \Delta T$ is in agreement with data for not too large values of $\Delta S$. This shows clearly that it can be misleading to make statements based on the $S$ parameter alone. Moreover the new contribution to $S$ can be decreased by taking into account a mass splitting of the fourth generation. Finally the SM4 can also compensate effects of a high mass of the Higgs boson. The authors of [88] state "the fit to electroweak data is in agreement with the existence of a fourth generation and a light Higgs about as well as the fit to the standard model alone with $m_{H}=115 \mathrm{GeV}$." So now the SM4 is not only not excluded, it is also not disfavored by the electroweak data. (This statement is not in agreement with, for example, the 2012 version of [ 83,89$]$, where it is still claimed that the SM4 is disfavoured compared to the SM. We do not try to settle this issue here.)

Similar arguments as the ones in [88] have actually been made previously from a Russian group [90-92] and also in $[93,94]$. But none of these papers managed to trigger so much interest in the community as [88].

There were, however, still some missing points in the investigation of the electroweak precision observables. First, treating the fourth generation lepton masses as free parameters allows, for example, easily also a degenerate fourth quark generation, see, for example, [15, 95]. In Figure 2 (taken from [15]) the allowed mass splitting of fourth generation fermions according to the constraints from the Peskin and Takeuchi parameters is shown. One clearly sees that mass degenerate quarks of the fourth family are not ruled out. Similar results were obtained, for example, in $[95,96]$. Second, also the full CKM dependence of the $T$ and $U$ parameters has to be included. This was first done in [95] and leads to the interesting result that even for fixed lepton masses now a degenerate fourth quark generation is possible. (The dominant CKM effects in the electroweak precision observables were investigated in $[97,98]$.) In Figure 3 a comparison of the electroweak fit without CKM mixing, that is setting by hand $V_{t b}=1=V_{t^{\prime} b^{\prime}}$, with the fit with the full CKM dependence is shown. For both cases the lepton masses are fixed. In the case of no CKM mixing (a commonly used assumption, which is, however, not justified by any principle!) one finds that degenerate quark masses are more or less excluded, while degeneracy is perfectly consistent with data, if CKM mixing is included. Allowing for both free lepton masses and CKM mixing one obtains the result from Figure 4. Hence, the degeneracy of the fourth generation is clearly not ruled out by electroweak precision observables. Similar results, like in Figure 4, were obtained in [96]. Third, instead of the Peskin and Takeuchi parameter it is of course desirable to use the full electroweak observables. An effective way to implement them was developed in [99]. This approach was applied in $[18,100]$ in combination with the data from Higgs searches and will be discussed below. The $S, T, U$ parameters were also investigated in $[101,102]$ for the case of the SM4.

A fourth generation of fermions can also have sizeable effects on flavor observables. First, the 3 times 3 CKM matrix will in general no longer be unitary-only the 4 times 4 CKM matrix has to fulfill unitarity. Thus, the current strong constraints on, for example, $V_{t d}, V_{t s}$ and $V_{t b}$ are no longer valid and their values can differ sizeably from the $S M$ values, obtained in CKM fits, see, for example, [103-105]. Second, the new heavy fermions contribute via loops in flavor changing neutral current processes like $b \rightarrow s \gamma$ or $B$ mixing. Since there were for quite some time several deviations between SM predictions and measurements of certain flavor observables, see, for example, [106], there were justified hopes that this 


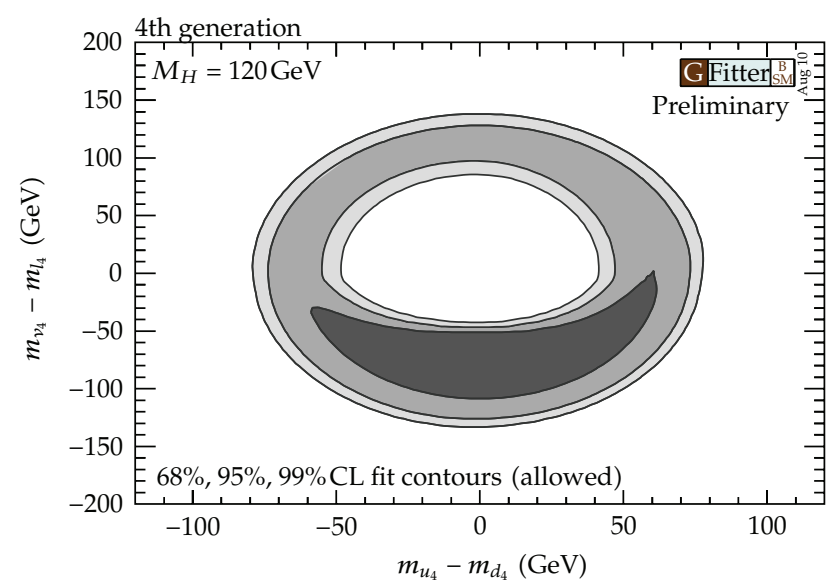

Figure 2: Allowed mass splitting of fourth generation fermions according to the constraints from electroweak precision observables. One clearly sees that mass degenerate quarks of the fourth family are not ruled out. Note, that for this result CKM mixing was neglected. Figure from [15].

might be the first hints for physics beyond the SM and this triggered a lot of interest in the literature, see, for example, the list of citations for [107]. Flavor observables within the SM4 were recently investigated in, for example, [108-119]. Because of the success of the CKM paradigm [120], one might expect that the CKM mixing between the first three generations and the fourth one is strongly constrained to tiny values. It was quite unexpected that flavor constraints alone allow still for huge effects in FCNC processes. In [109] it is explained in detail how for example, a $t^{\prime}$-contribution to the box diagram for the mass difference in the neutral $B$-meson system, which is larger than the SM value, can be compensated by the modified values of the CKM elements $V_{t d}, V_{t s}$, and to a lesser extent $V_{t b}$. Also quite unexpected was the fact that stronger bounds on the CKM mixing with a fourth generation of quarks are actually obtained from electroweak precision observables [95, 97, 98]. Nevertheless there is a lot room for new effects in the CKM sector of the SM4, which might explain the above-mentioned discrepancies in the flavor sector. Unfortunately now many of these discrepancies have disappeared because of new data, in particular from $\mathrm{LHCb}$ see, for example, $[121,122]$. Also a long standing discrepancy concerning the leptonic decay $B \rightarrow \tau v$ seems to have been resolved experimentally by the Belle Collaboration [123].

To summarize the results of this section we make the following statement: the SM4 can easily accommodate all flavor and electroweak data and the previous claims on the exclusion of the SM4 relied on unjustified additional assumptions. One thing to keep in mind is the fact that currently twoloop electroweak corrections for the electroweak precision observables are still missing and these corrections might be sizeable.

Currently there are also stringent limits on the masses of the fourth generation quarks from direct searches [124129], which push the values of the masses close to the nonperturbative regime. Here we only would like to mention that many of these analyses rely on some additional, nongeneral assumptions, for example, about the CKM mixing of the fourth generation [130]. Giving up these additional assumptions one gets typically considerably weaker bounds on the masses.

Till now we found that the SM4 is resistant against all experimental attacks. In the next section we will, however, show that the SM4 cannot comply with the recent data from Higgs searches.

2.3. Common Folklore. Concerning Higgs production and Higgs decay the common folklore reads as follows: gluon fusion is enhanced by a factor of nine (e.g., [131]) due to the additional running of the $t^{\prime}$ - and $b^{\prime}$ - quark in the loop of Figure 1 and therefore the SM4 can easily be killed, if no huge Higgs signals are observed. On the other hand in the SM4 severe cancellations arise in the decay $H \rightarrow \gamma \gamma$ [132-135]. In (13) of [88] the situation for a light Higgs mass (i.e., below $200 \mathrm{GeV}$ ) was roughly summarized as

$$
\begin{aligned}
& \left.\sigma(g g \longrightarrow H) \cdot \operatorname{Br}(H \longrightarrow \gamma \gamma)\right|_{\mathrm{SM} 4} \\
& \left.\quad \approx \sigma(g g \longrightarrow H) \cdot \operatorname{Br}(H \longrightarrow \gamma \gamma)\right|_{\mathrm{SM}}, \\
& \left.\sigma(g g \longrightarrow H) \cdot \operatorname{Br}(H \longrightarrow Z Z)\right|_{\mathrm{SM} 4} \\
& \left.\quad \approx(5 \ldots 8) \sigma(g g \longrightarrow H) \cdot \operatorname{Br}(H \longrightarrow Z Z)\right|_{\mathrm{SM}}, \\
& \left.\sigma(g g \longrightarrow H) \cdot \operatorname{Br}(H \longrightarrow f \bar{f})\right|_{\mathrm{SM} 4} \\
& \left.\quad \approx 5 \sigma(g g \longrightarrow H) \cdot \operatorname{Br}(H \longrightarrow f \bar{f})\right|_{\mathrm{SM}} .
\end{aligned}
$$

References $[136,137]$ obtain higher values for $g g \rightarrow H \rightarrow$ $\gamma \gamma$-there the SM4 value is enhanced by a factor of up to 4 compared to the SM value. Reference [138] gives only values for $M_{H}$ between $115 \mathrm{GeV}$ and $130 \mathrm{GeV}$ and there the enhancement factor for $g g \rightarrow H \rightarrow \gamma \gamma$ is between 1.4 and 2.6 .

Despite these minor differences there is a common result: in the SM4 one expects more or at least as much Higgs events (depending on the channel) as in the SM. Hence, seeing nothing above the SM expectation rules out the SM4. These line of arguments can, for example, be found in the interpretation of the LHC Higgs searches presented at Lepton-Photon 2011, see also [139].

There are, however, two possible flaws in this argumentation, which have to be taken into account and which might actually revert the conclusions.

2.4. Loop Hole No.1: Invisible Higgs Decays. First, there is still the possibility that the heavy fourth neutrino is lighter than half of the Higgs mass. Current experimental bounds do not exclude a fourth neutrino with a mass larger than half of the $Z$ mass (depending on the lifetime of the fourth neutrino). Having a neutrino with $m_{v_{4}}<m_{H} / 2$ would open up a new invisible decay channel, which can suppress all other Higgs branching ratios and therefore compensate for the enhancement factor of the gluon fusion process within the SM4. This idea was first presented in [140] and later on further 


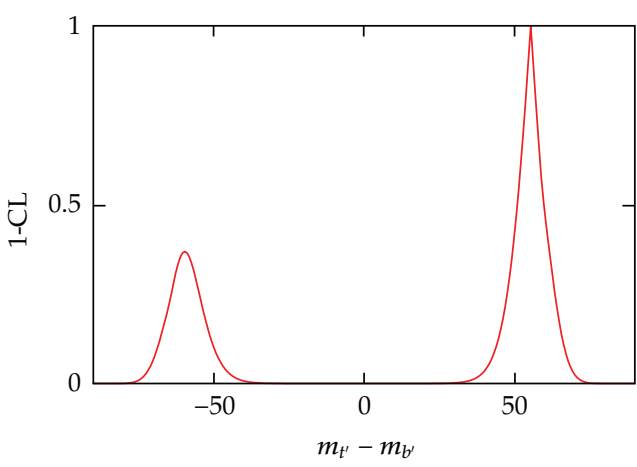

(a)

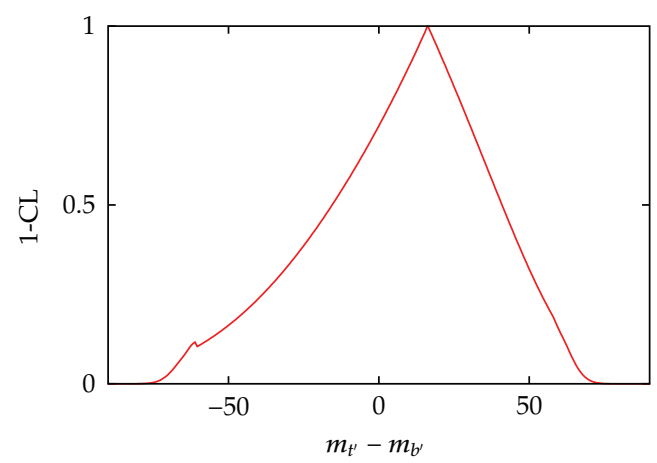

(b)

FIGURE 3: Allowed mass splitting of fourth generation quarks according to the constraints from electroweak precision observables. (a) CKM mixing is forced to be zero, (b) it is allowed to an extent that does not violate any bound from flavor physics. For both pictures the lepton masses were fixed.

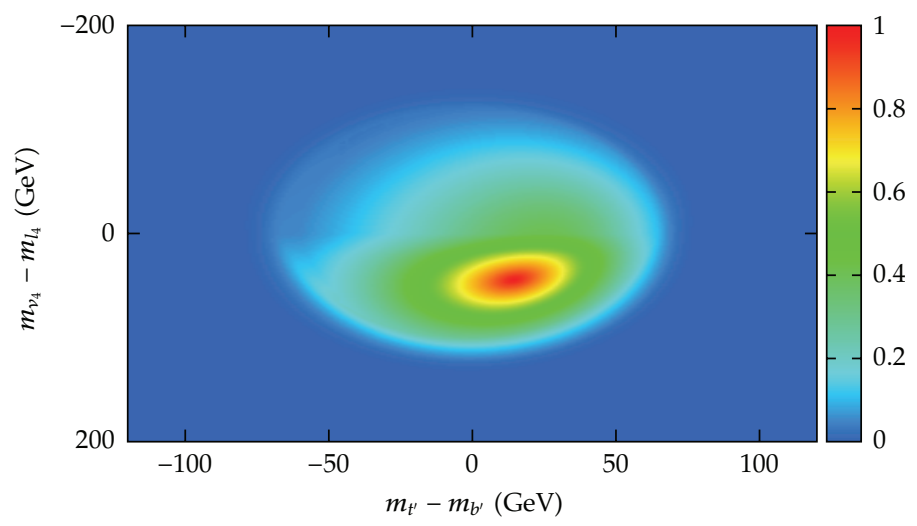

FIGURE 4: Allowed mass splitting of fourth generation fermions according to the constraints from electroweak precision observables, allowing for CKM mixing and free lepton masses.

investigated in, for example, [141-145]. A more general lepton sector, where the neutrino can also have a Majorana character, was discussed, for example, in [146-149].

2.5. Loop Hole No.2: Huge NLO Electroweak Corrections. NLO-electroweak corrections to the Higgs production have already been determined in the midnineties [38-40]. In these works the dominant contributions proportional to $G_{F} m_{t}^{2}$ were determined. The full dependence on the mass of the heavy fermions was calculated recently in $[16,150]$. As a result it turned out that the corrections can be huge. The corrections to the gluon fusion process are shown in Figure 5. For low Higgs masses $(100 \mathrm{GeV}-200 \mathrm{GeV})$ the NLO-electroweak corrections are sizeable-of the order of $+10 \%$. For large Higgs masses $(>900 \mathrm{GeV})$ they are huge, that is, larger than $-50 \%$ and the perturbative approach probably breaks down.

Also the decay rates of the Higgs boson are sizeably affected by NLO-electroweak corrections. In Figure 6 a comparison of the different Higgs branching ratios within the
SM4 and the standard model is shown. The invisible decay of the Higgs boson is not yet taken into account here.

With the results from Figures 5 and 6 one obtains the following expectations for Higgs searches:

$$
\begin{aligned}
& \sigma(g g\longrightarrow H)\left.\cdot \mathrm{Br}(H \longrightarrow \gamma \gamma)\right|_{\mathrm{SM} 4} \\
&<\left.0.27 \sigma(g g \longrightarrow H) \cdot \mathrm{Br}(H \longrightarrow \gamma \gamma)\right|_{\mathrm{SM}}, \\
&\approx 1) \\
& \sigma(g g\longrightarrow H)\left.\cdot \mathrm{Br}(H \longrightarrow Z Z)\right|_{\mathrm{SM} 4} \\
&\left.\approx(1.35 \ldots 9) \sigma(g g \longrightarrow H) \cdot \mathrm{Br}(H \longrightarrow Z Z)\right|_{\mathrm{SM}}{ }^{\prime} \\
& \approx(5 \ldots 8) \\
& \sigma(g g\longrightarrow H)\left.\cdot \mathrm{Br}(H \longrightarrow b \bar{b})\right|_{\mathrm{SM} 4} \\
&\left.\approx(6.3 \ldots 27) \sigma(g g \longrightarrow H) \cdot \mathrm{Br}(H \longrightarrow b \bar{b})\right|_{\mathrm{SM}^{\prime}} \\
&(\approx 5) .
\end{aligned}
$$




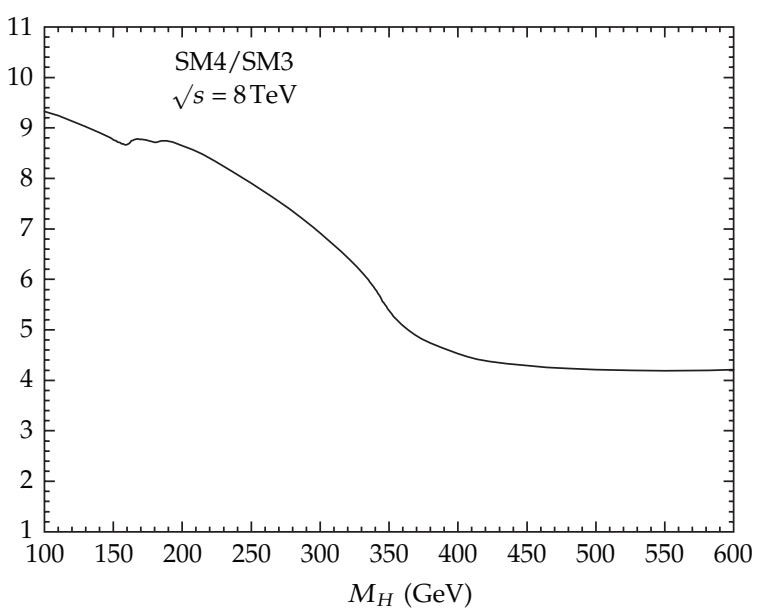

FIgURE 5: Comparison of gluon fusion cross-section within the SM4 and the standard model. Depending on the value of the Higgs mass sizeable deviations of the naive expectation for ratio to be nine is possible. For Higgs masses in the region of $125 \mathrm{GeV}$ this expectation works, however, very well. Figure from [16].

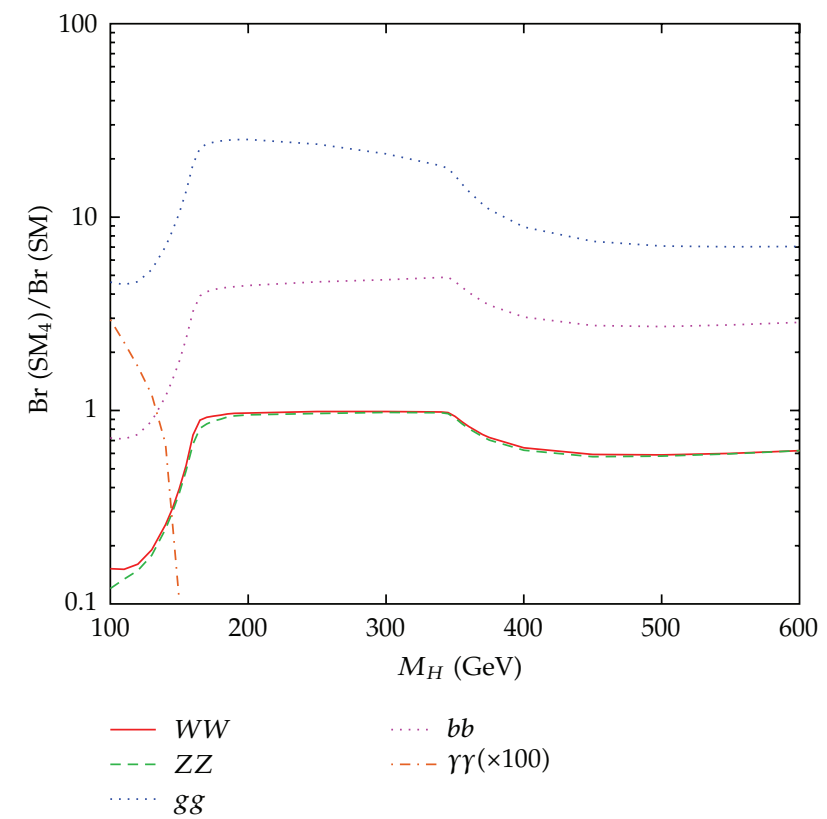

FIgURE 6: Comparison of Higgs decay rates within the SM4 and the standard model including NLO-electroweak corrections. In particular interesting is the strong reduction of the $H \rightarrow \gamma \gamma$ channel. Figure from [16].

For a comparison we have also shown the results from [88] (see (5)) in brackets below the new values. To summarize, NLO-electroweak corrections turned out to be huge and therefore any analysis without these corrections, that is, any analysis before 2012, cannot provide reliable results. The most stringent new result is now the predicted underproduction in the channel $H \longrightarrow \gamma \gamma$.

There are however still some minor drawbacks, which should be kept in mind. First in the calculation of the
NLO-electroweak corrections [16, 150] CKM mixing was neglected. Second the results of $[16,150]$ are not given for the general parameter space of the SM4. The parameter range was considerably extended from $\mathrm{vl}$ (only one parameter point) of [16] to v2, but still, for example, the interesting region with a neutrino mass of about $60 \mathrm{GeV}$ and a corresponding lepton mass, which is consistent with electroweak precision observables, is not available. Approximate formulas for this mass region are, however, included in the latest version (4.45) of the programme HDECAY [151, 152], which became publically available on March 13, 2012.

2.6. Closing the Loop Holes. The complete NLO-electroweak corrections to Higgs production and decay and the possibility of an invisible Higgs decay were taken into account for the first time in [17] and later on in $[18,100,153,154]$. To the best of my knowledge in experimental investigations at ATLAS [155] and CMS [156] the NLO-electroweak corrections are now included but the possibility of a fourth neutrino in which the Higgs could decay is still not considered. After Moriond 2012 all theoretical tools, in particular the NLO-electroweak corrections, were publically available and now one was for the first time in a position where definite conclusions can be drawn. Assuming a Higgs mass of $125 \mathrm{GeV}$ one gets an enhancement factor of about nine for gluon fusion and the Higgs branching ratios given in Figure 7 in dependence of the mass of the fourth neutrino. For high neutrino masses one obtains the classic result that the SM4 predicts too many Higgs events and hence, can be excluded if no more Higgs events than expected in the SM are found. For low masses of the fourth neutrinos the SM4 yields, however, less Higgs events than in the SM. In Figure 7 still an approximate NLO expression for $H \rightarrow \gamma \gamma$ was used. The full expression will give a further suppression of the two-photon channel by a factor of about six, see [17]. For neutrino masses between $100 \mathrm{GeV}$ and $600 \mathrm{GeV}$ and for a Higgs mass of $125 \mathrm{GeV}$ one gets now [17]

$$
\begin{aligned}
\sigma(g g & \longrightarrow H)\left.\cdot \operatorname{Br}(H \longrightarrow \gamma \gamma)\right|_{\mathrm{SM} 4} \\
& \left.\approx(0.09 \ldots 0.2) \sigma(g g \longrightarrow H) \cdot \operatorname{Br}(H \longrightarrow \gamma \gamma)\right|_{\mathrm{SM}} .
\end{aligned}
$$

This clearly means that if a SM-like Higgs signal in the twophoton channel is seen at LHC then the SM4 is ruled out because there at most $20 \%$ of the events expected in the SM can be created. Thus, contrary to the common belief, the SM4 might actually be killed because of a predicted underproduction of Higgses and not of an overproduction. Due to the huge cancellations that arise now in the decay $H \rightarrow \gamma \gamma$, one might, however query the reliability of the theory prediction for $\Gamma(H \rightarrow \gamma \gamma)$. In Moriond Tevatron also presented results [66] for $p+\bar{p} \rightarrow H+V \rightarrow b \bar{b}+V$, which were within the statistical uncertainties in agreement with the SM expectations. In the SM4 one expects at most $35 \%$ of the number of SM events [17]. The conclusion of [17] was that if the currents hint for Higgs boson production and decay in the channels $H \rightarrow \gamma \gamma$ and $H \rightarrow b \bar{b}$ presented in Moriond 2012 will stay with more data at their current values, then the SM4 


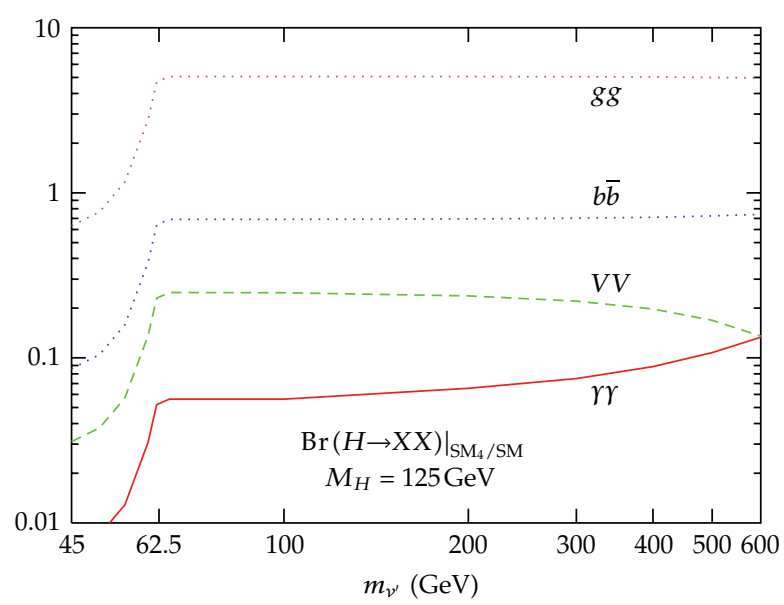

FIGURE 7: Higgs branching ratios in dependence of the mass of the fourth neutrino for a Higgs mass of $125 \mathrm{GeV}$. Below $m_{v}^{\prime}=62.5 \mathrm{GeV}$ the new invisible decay channel $H \rightarrow v_{4} v_{4}$ opens and all other branching ratios will be strongly suppressed. This plot was, however, created with an approximate NLO expression for $H \rightarrow \gamma \gamma$. The full expression will give a further suppression of the two-photon channel by a factor of about six. Figure taken from [17].

will be excluded, because it predicts considerably less events in these two channels.

A combined fit of electroweak precision observables and data for Higgs boson production and decay was presented in [100]. Later on this was updated, in particular with data for the channel $H \rightarrow \tau^{+} \tau^{-}$in [18]. In Figure 8 the pulls of the Higgs signal strengths are shown for the channels $H \rightarrow \gamma \gamma, W W, Z Z, b \bar{b}$, and $\tau^{+} \tau^{-}$, comparing the SM3 and the SM4 predictions. It turns out that the biggest effect is seen in the channel $H \rightarrow \tau^{+} \tau^{-}$. Here the classic expectations holds: the SM4 predicts a much higher rate than observed. For the channels $H \rightarrow \gamma \gamma$ and $H \rightarrow b \bar{b}$ the reverse effect arises: the SM4 predicts a much lower rate than observed experimentally. One can see from Figure 8 that also the SM does not describe the data perfectly: the data for $H \rightarrow \gamma \gamma$ and $H \rightarrow b \bar{b}$ are higher than expected, while the data for $H \rightarrow W W$ and $H \rightarrow \tau^{+} \tau^{-}$are lower than expected. But all in all the SM describes the data much better than the SM4. This will be quantified below.

In Figure 9 the best fit results in dependence of the mass of the fourth neutrino are shown. The SM4 has only a small chance to be viable in regions where the Higgs boson can decay invisibly in the two neutrinos. This is strongly triggered by the data for $H \rightarrow \tau^{+} \tau^{-}$; in this channel the SM4 predicts a strong enhancement, which can only be compensated by the invisible decay of the Higgs boson. To be more quantitative: before the ICHEP 2012 Conference the SM4 was disfavored compared to the SM by 3.1 standard deviations [18]. (For more information about how the corresponding $P$ value of this exclusion can be determined, see [157].)

2.7. Welcome to the Real World. On July 4, 2012 ATLAS and CMS announced new results for the Higgs boson search [14].
The new data worsens the situation for the SM4 in several points.

(1) Both ATLAS and CMS see a $H \rightarrow \gamma \gamma$-signal with a statistical significance of more than 4 standard deviations. The total observed rate was higher than expected by the SM (a factor of $1.9 \pm 0.5$ for ATLAS and a factor of $1.56 \pm 0.43$ for CMS). In the SM4 one would expect instead a reduction of the rate by at least a factor of 5 compared to the standard model, see (7). Thus, both ATLAS and CMS individually see a $H \rightarrow \gamma \gamma$-signal, which is about 4 standard deviations away from the expectation of the SM4, which rules out the SM4. As discussed above, the theory prediction for $H \rightarrow \gamma \gamma$ in the SM4 suffers from severe cancellations, so one might not want to rely on this decay channel alone.

(2) On July, 2012 also CDF and D0 updated their Higgs search [67] in the Higgs-strahlung channel, discussed above. There the statistical significance increased from 2.6 standard deviations in [66] to 2.9 standard deviations in [67]. At $m_{H}=125 \mathrm{GeV}$ Tevatron finds a signal strength of $1.97_{-0.68}^{+0.74}$, so a little above the SM expectation, while the SM4 predicts values below 0.35 [17]. Again a stronger indication against the SM4 compared to the status of Moriond 2012.

(3) In the SM4 one would expect a sizeable enhancement of the $H \rightarrow \tau \tau$ channel, see for example, [18], which is not observed [14] in the new data. A further argument against the SM4 at the $4 \sigma$ level.

In v2 of [17] the following conclusion was drawn from these new results: "...we conclude that the standard model with a perturbative 4th generation and one Higgs doublet is ruled out by this new experimental developments."

Before these new results the SM4 was excluded by 3.1 standard deviations; with the new data we expect the SM4 to be excluded by about five standard deviations [158].

\section{Beyond the Simple Perturbative SM4}

The results, which were discussed here, relied strongly on the assumption of perturbativity of the heavy fermions and are also only valid for a minimal Higgs sector, that is, for only one Higgs doublet.

3.1. NonPerturbative SM4. Investigations of a nonperturbative fourth generation can be found, for example, in [159].

3.2. Extended Higgs Sectors. The easiest way to avoid the above-discussed bounds on an additional fermion generation is to extend also the sector. A fourth generation in combination with a second Higgs doublet has been discussed in $[93,160-166]$. 


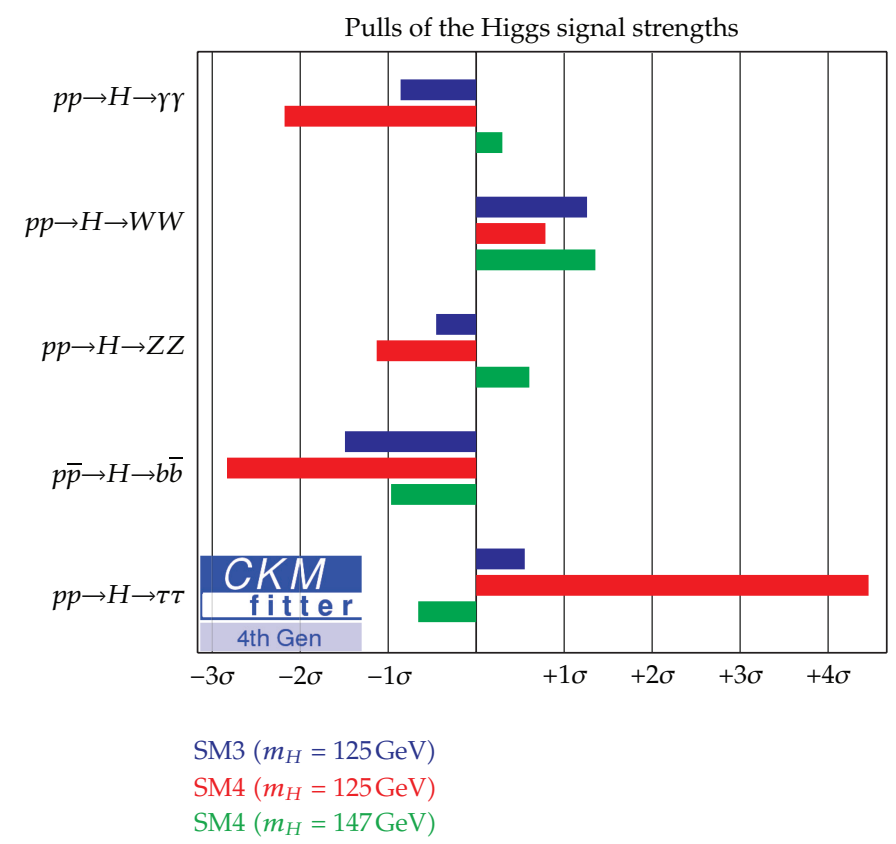

FIGURE 8: Pulls of the Higgs signal strengths with the data before ICHEP 2012. Figure from [18].

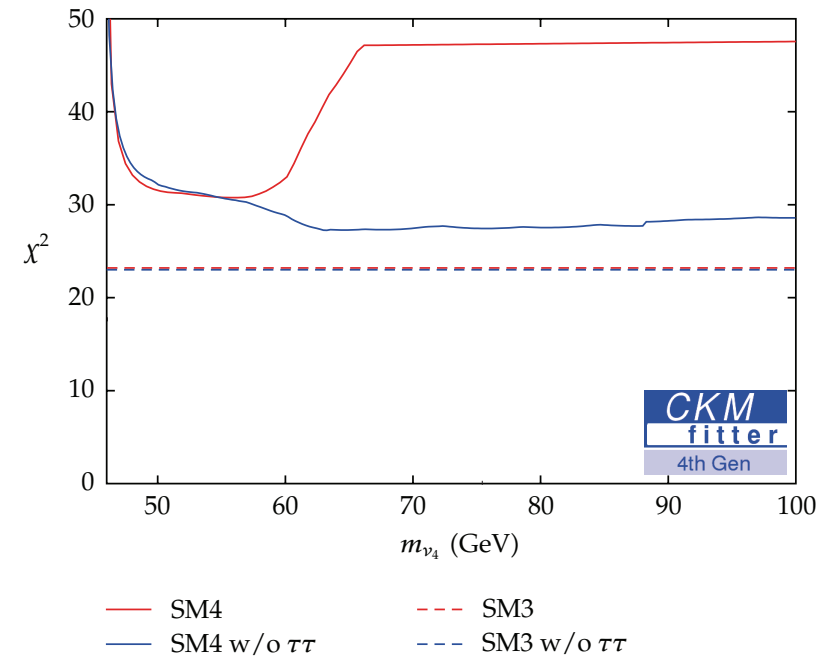

FIGURE 9: Best fit results in dependence of the mass of the fourth neutrino. As soon as $H \rightarrow \tau^{+} \tau^{-}$is included, the only remaining possibility for the SM4 is to open up the decay channel $H \rightarrow v_{4} v_{4}$. Figure from [18].

\section{Discussions and Conclusions}

We reviewed the past and current status of the extension of the SM by an additional chiral generation of fermions. An amusing aspect thereby was the fact that the SM4 was declared dead many times. But all these declarations relied on some unjustified assumptions-giving them up the model was still viable. This clearly would be an interesting topic for a sociological study: why were there so many prejudices against the existence of this simple model? Nevertheless, it turned finally out that the SM4 is ruled out by experimental data (So unfortunately we did not learn the lesson that prejudices should be avoided in physics.): The SM4 cannot accommodate the new data for Higgs searches presented by LHC and Tevatron and thus, it is excluded.

There are still some missing ingredients like that the 2loop corrections to the electroweak precision observables within the SM4 are still missing. Moreover the NLOelectroweak corrections to Higgs production and decay were calculated by neglecting CKM mixing.

Finally we commented very briefly that extending in addition the Higgs sector, for example, by an additional Higgs doublet might circumvent many of the above-discussed bounds.

\section{Acknowledgments}

The author thanks Markus Bobrowski, Otto Eberhardt, Abdelhak Djouadi, Geoffrey Herbert, Heiko Lacker, Andreas Menzel, Uli Nierste, Johann Riedl, Jürgen Rohrwild, Heinrich Päs [167], Dario Schalla, and Martin Wiebusch for the pleasant collaboration on topics related to a fourth generation of fermions. He is supported by the DFG via the Heisenberg Programme and also via the Grant LE 1246/9-1.

\section{References}

[1] S. L. Glashow, "Partial-symmetries of weak interactions," Nuclear Physics, vol. 22, no. 4, pp. 579-588, 1961.

[2] S. Weinberg, "A model of Leptons," Physical Review Letters, vol. 19, no. 21, pp. 1264-1266, 1967.

[3] F. Englert and R. Brout, "Broken symmetry and the mass of gauge vector mesons," Physical Review Letters, vol. 13, no. 9, pp. 321-323, 1964. 
[4] P. W. Higgs, "Broken symmetries, massless particles and gauge fields," Physics Letters, vol. 12, no. 2, pp. 132-133, 1964.

[5] P. W. Higgs, "Broken symmetries and the masses of gauge bosons," Physical Review Letters, vol. 13, no. 16, pp. 508-509, 1964.

[6] G. S. Guralnik, C. R. Hagen, and T. W. B. Kibble, "Global conservation laws and massless particles," Physical Review Letters, vol. 13, no. 20, pp. 585-587, 1964.

[7] P. W. Higgs, "Spontaneous symmetry breakdown without massless bosons," Physical Review, vol. 145, no. 4, pp. 1156-1163, 1966.

[8] N. Cabibbo, "Unitary symmetry and leptonic decays," Physical Review Letters, vol. 10, no. 12, pp. 531-533, 1963.

[9] M. Kobayashi and T. Maskawa, "CP-violation in the renormalizable theory of weak interaction," Progress of Theoretical Physics, vol. 49, no. 2, pp. 652-657, 1973.

[10] A. D. Sakharov, "Violation of CP symmetry, C-asymmetry and baryon asymmetry of the Universe," Pis'ma $v$ Zhurnal Eksperimental'noi i Teoreticheskoi Fiziki, vol. 5, pp. 32-35, 1967, translation in JETP Letters, vol. 5, pp. 24-27, 1967.

[11] A. D. Sakharov, "Violation of CP in variance, C asymmetry, and baryon asymmetry of the universe," Soviet Physics Uspekhi, vol. 34 , no. 5, article 392, 1991.

[12] A. D. Sakharov, Uspekhi Fizicheskikh Nauk, vol. 161, p. 61, 1991.

[13] W. S. Hou, "Source of CP violation for baryon asymmetry of the Universe," Chinese Journal of Physics, vol. 47, p. 134, 2009, http://arxiv.org/abs/0803.1234.

[14] http://indico.cern.ch/conferenceDisplay.py?confld=197461.

[15] M. Baak, M. Goebel, J. Haller et al., "Updated status of the global electroweak fit and constraints on new physics," European Physical Journal C, vol. 72, no. 5, article 2003, 2012.

[16] A. Denner, S. Dittmaier, A. Mück et al., "Higgs production and decay with a fourth standard-model-like fermion generation," European Physical Journal C, vol. 72, no. 5, pp. 1-15, 2012.

[17] A. Djouadi and A. Lenz, "Sealing the fate of a fourth generation of fermions," Physics Letters B, vol. 715, no. 4-5, pp. 310-314, 2012.

[18] O. Eberhardt, A. Lenz, A. Menzel, U. Nierste, and M. Wiebusch, "Status of the fourth fermion generation before ICHEP2012: Higgs data and electroweak precision observables," Physical Review D, vol. 86, no. 7, Article ID 074014, 5 pages, 2012.

[19] H. M. Georgi, S. L. Glashow, M. E. MacHacek, and D. V. Nanopoulos, "Higgs bosons from two-gluon annihilation in proton-proton collisions," Physical Review Letters, vol. 40, no. 11, pp. 692-694, 1978.

[20] Figure taken from the homepage of Robert Harlander, http://particle.uni-wuppertal.de/harlander/research/ggh/.

[21] A. Djouadi, "The anatomy of electroweak symmetry breaking: tome I: the Higgs boson in the standard model," Physics Reports, vol. 457, no. 1-4, pp. 1-216, 2008.

[22] A. Djouadi, M. Spira, and P. M. Zerwas, "Production of Higgs bosons in proton colliders. QCD corrections," Physics Letters B, vol. 264, no. 3-4, pp. 440-446, 1991.

[23] S. Dawson, "Radiative corrections to Higgs boson production," Nuclear Physics B, vol. 359, no. 2-3, pp. 283-300, 1991.

[24] M. Spira, A. Djouadi, D. Graudenz, and P. M. Zerwas, "Higgs boson production at the LHC," Nuclear Physics B, vol. 453, no. 1-2, pp. 17-82, 1995.

[25] R. V. Harlander, "Virtual corrections to gg $\rightarrow \mathrm{H}$ to two loops in the heavy top limit," Physics Letters B, vol. 492, no. 1-2, pp. 7480,2000 .
[26] S. Catani, D. de Florian, and M. Grazzini, "Higgs production in hadron collisions: soft and virtual QCD corrections at NNLO," Journal of High Energy Physics, vol. 2001, no. 5, article 25, 2001.

[27] R. V. Harlander and W. B. Kilgore, "Soft and virtual corrections to $\mathrm{pp} \rightarrow \mathrm{H}+\mathrm{X}$ at next-to-next-to-leading order," Physical Review $D$, vol. 64, no. 1, Article ID 013015, 9 pages, 2001.

[28] R. V. Harlander and W. B. Kilgore, "Next-to-next-to-leading order Higgs production at Hadron colliders," Physical Review Letters, vol. 88, no. 20, Article ID 201801, 4 pages, 2002.

[29] C. Anastasiou and K. Melnikov, "Higgs boson production at hadron colliders in NNLO QCD," Nuclear Physics B, vol. 646, no. 1-2, pp. 220-256, 2002.

[30] V. Ravindran, J. Smith, and W. L. van Neerven, "NNLO corrections to the total cross section for Higgs boson production in hadron-hadron collisions," Nuclear Physics B, vol. 665, pp. 325366, 2003.

[31] S. Catani, D. de Florian, M. Grazzini, and P. Nason, "Soft-gluon resummation for Higgs boson production at hadron colliders," Journal of High Energy Physics, vol. 2003, no. 7, article 28, 2003.

[32] S. Marzani, R. D. Ball, V. Del Duca, S. Forte, and A. Vicini, "Finite-top-mass effects in NNLO Higgs production," Nuclear Physics B, vol. 186, pp. 98-101, 2009.

[33] R. V. Harlander and K. J. Ozeren, “Top mass effects in Higgs production at next-to-next-to-leading order QCD: virtual corrections," Physics Letters B, vol. 679, no. 5, pp. 467-472, 2009.

[34] A. Pak, M. Rogal, and M. Steinhauser, "Virtual three-loop corrections to Higgs boson production in gluon fusion for finite top quark mass," Physics Letters B, vol. 679, no. 5, pp. 473-477, 2009.

[35] R. V. Harlander and K. J. Ozeren, "Finite top mass effects for hadronic Higgs production at next-to-next-to-leading order," Journal of High Energy Physics, vol. 2009, no. 11, article 088, 2009.

[36] A. Pak, M. Rogal, and M. Steinhauser, "Finite top quark mass effects in NNLO Higgs boson production at LHC," Journal of High Energy Physics, vol. 2010, no. 2, article 25, 2010.

[37] R. V. Harlander, H. Mantler, S. Marzani, and K. J. Ozeren, "Higgs production in gluon fusion at next-to-next-to-leading order QCD for finite top mass," The European Physical Journal C, vol. 66, no. 3-4, pp. 359-372, 2010.

[38] A. Djouadi and P. Gambino, "Leading electroweak correction to Higgs boson production at proton colliders," Physical Review Letters, vol. 73, no. 19, pp. 2528-2531, 1994.

[39] A. Djouadi, P. Gambino, and B. A. Kniehl, "Two-loop electroweak heavy-fermion corrections to Higgs-boson production and decay," Nuclear Physics B, vol. 523, no. 1-2, pp. 17-39, 1998.

[40] F. Fugel, B. A. Kniehl, and M. Steinhauser, "Two-loop electroweak correction of $\mathrm{O}\left(\mathrm{G}_{F} M_{t}^{2}\right)$ to the Higgs-boson decay into photons," Nuclear Physics B, vol. 702, no. 1-2, pp. 333-345, 2004.

[41] G. Degrassi and F. Maltoni, "Two-loop electroweak corrections to Higgs production at hadron colliders," Physics Letters B, vol. 600, no. 3-4, pp. 255-260, 2004.

[42] U. Aglietti, R. Bonciani, G. Degrassi, and A. Vicini, “Two-loop light fermion contribution to Higgs production and decays," Physics Letters B, vol. 595, no. 1-4, pp. 432-441, 2004.

[43] S. Actis, G. Passarino, C. Sturm, and S. Uccirati, "NLO electroweak corrections to Higgs boson production at hadron colliders," Physics Letters B, vol. 670, no. 1, pp. 12-17, 2008.

[44] C. Anastasiou, R. Boughezal, and F. Petriello, "Mixed QCDelectroweak corrections to Higgs boson production in gluon fusion," Journal of High Energy Physics, vol. 2009, no. 4, article 3, 2009. 
[45] O. Brein, A. Djouadi, and R. Harlander, "NNLO QCD corrections to the Higgs-strahlung processes at hadron colliders," Physics Letters B, vol. 579, no. 1-2, pp. 149-156, 2004.

[46] O. Brein, R. Harlander, M. Wiesemann, and T. Zirke, "Topquark mediated effects in hadronic Higgs-Strahlung," The European Physical Journal C, vol. 72, no. 2, article 1868, 2012.

[47] R. V. Harlander, J. Vollinga, and M. M. Weber, "Gluon-induced weak boson fusion," Physical Review D, vol. 77, no. 5, Article ID 053010, 6 pages, 2008.

[48] G. Aad, T. Abajyan, B. Abbott et al., "Combined search for the standard model Higgs boson in pp collisions at $\sqrt{s}=7 \mathrm{TeV}$ with the ATLAS detector," Physical Review D, vol. 86, no. 3, Article ID 032003, 31 pages, 2012.

[49] G. Aad, T. Abajyan, B. Abbott et al., "Search for the standard model Higgs boson produced in association with a vector boson and decaying to a b-quark pair with the ATLAS detector," Physics Letters B. http://arxiv.org/abs/1207.0210.

[50] G. Aad, T. Abajyan, B. Abbott et al., "Search for the Higgs boson in the $\mathrm{H} \rightarrow \mathrm{WW} \rightarrow \operatorname{lnujj}$ decay channel at $\sqrt{(s)}=7 \mathrm{TeV}$ with the ATLAS detector," Physics Letters $B$. http://arxiv.org/abs/1206.6074.

[51] G. Aad, T. Abajyan, B. Abbott et al., "Search for the standard model Higgs boson in the $\mathrm{H}$ to tau+ tau- decay mode in $\sqrt{(s)}=$ $7 \mathrm{TeV}$ pp collisions with ATLAS," Journal of High Energy Physics. http://arxiv.org/abs/1206.5971.

[52] G. Aad, T. Abajyan, B. Abbott et al., "Search for a standard model Higgs boson in the mass range $200-600 \mathrm{GeV}$ in the $\mathrm{H} \rightarrow \mathrm{ZZ} \rightarrow$ llqq decay channel," Physics Letters $B$. http://arxiv.org/abs/1206.2443.

[53] G. Aad, T. Abajyan, B. Abbott et al., "Search for the standard model Higgs boson in the $\mathrm{H} \rightarrow \mathrm{WW}(*) \rightarrow \mathrm{l}$ nu $\mathrm{l}$ nu decay mode with $4.7 / \mathrm{fb}$ of ATLAS data at $\sqrt{(s)}=7 \mathrm{TeV}$, Physics Letters $B$, vol. 716, no. 1, pp. 62-81, 2012.

[54] G. Aad, T. Abajyan, B. Abbott et al., "Search for a standard model Higgs boson in the $\mathrm{H} \rightarrow \mathrm{ZZ} \rightarrow$ llnunu decay channel using 4.7 $\mathrm{fb}^{-1}$ of $\sqrt{(s)}=7 \mathrm{TeV}$ data with the ATLAS detector," Physics Letters B. http://arxiv.org/abs/1205.6744.

[55] G. Aad, T. Abajyan, B. Abbott et al., "Search for the standard model Higgs boson in the decay channel $\mathrm{H} \rightarrow \mathrm{ZZ}\left({ }^{*}\right)$-4l with 4.8 $\mathrm{fb}^{-1}$ of pp collision data at $\sqrt{(s)}=7 \mathrm{TeV}$ with ATLAS," Physics Letters B, vol. 710, no. 3, pp. 383-402, 2012.

[56] G. Aad, T. Abajyan, B. Abbott et al., "Search for the standard model Higgs boson in the diphoton decay channel with $4.9 \mathrm{fb}^{-1}$ of pp collision data at $\sqrt{s}=7 \mathrm{TeV}$ with ATLAS," Physical Review Letters, vol. 108, no. 11, Article ID 111803, 19 pages, 2012.

[57] S. Chatrchyan, V. Khachatryan, A.M. Sirunyan et al., "Search for the standard model Higgs boson decaying to bottom quarks in pp collisions at $\sqrt{s}=7 \mathrm{TeV}$," Physics Letters B, vol. 710, no. 2, pp. 284-306, 2012.

[58] S. Chatrchyan, V. Khachatryan, A.M. Sirunyan et al., "Search for neutral Higgs bosons decaying to tau pairs in pp collisions at $\sqrt{s}=7$ TeV," Physics Letters B, vol. 713, no. 2, pp. 68-90, 2012.

[59] S. Chatrchyan, V. Khachatryan, A.M. Sirunyan et al., "Search for the standard model Higgs boson in the $\mathrm{H} \rightarrow \mathrm{ZZ} \rightarrow \ell^{+} \ell^{-} \tau^{+} \tau^{-}$ decay channel in pp collisions at $\sqrt{s}=7 \mathrm{TeV}$,' Journal of High Energy Physics, vol. 2012, no. 3, article 81, 2012.

[60] S. Chatrchyan, V. Khachatryan, A.M. Sirunyan et al., "Search for the standard model Higgs boson in the $\mathrm{H} \rightarrow \mathrm{ZZ} \rightarrow 2 \ell 2 v$ channel in pp collisions at $\sqrt{s}=7 \mathrm{TeV}$," Journal of High Energy Physics, vol. 2012, no. 3, article 40, 2012.
[61] S. Chatrchyan, V. Khachatryan, A.M. Sirunyan et al., "Search for the standard model Higgs boson in the decay channel $\mathrm{H} \rightarrow$ $\mathrm{ZZ} \rightarrow 4 \mathrm{l}$ in pp Collisions at $\sqrt{s}=7 \mathrm{TeV}$," Physical Review Letters, vol. 108, no. 11, Article ID 111804, 17 pages, 2012.

[62] S. Chatrchyan, V. Khachatryan, A.M. Sirunyan et al., "Search for the standard model Higgs boson decaying to $\mathrm{W}^{+} \mathrm{W}^{-}$in the fully leptonic final state in pp collisions at $\sqrt{s}=7 \mathrm{TeV}$," Physics Letters B, vol. 710, no. 1, pp. 91-113, 2012.

[63] S. Chatrchyan, V. Khachatryan, A.M. Sirunyan et al., "Combined results of searches for the standard model Higgs boson in pp collisions at $\sqrt{s}=7 \mathrm{TeV}$," Physics Letters $B$, vol. 710, no. 1, pp. 26-48, 2012.

[64] S. Chatrchyan, V. Khachatryan, A.M. Sirunyan et al., "Search for the standard model Higgs boson decaying into two photons in pp collisions at $\sqrt{s}=7 \mathrm{TeV}$,' Physics Letters $B$, vol. 710, no. 3, pp. 403-425, 2012.

[65] S. Chatrchyan, V. Khachatryan, A.M. Sirunyan et al., "Search for a Higgs boson in the decay channel $\mathrm{H}$ to $\mathrm{ZZ}\left({ }^{*}\right)$ to q qbar $\mathrm{l}-\mathrm{l}+$ in pp collisions at $\sqrt{s}=7 \mathrm{TeV}$,' Journal of High Energy Physics, vol. 2012, no. 4, article 36, 2012.

[66] W. Fisher, (CDF and D0), Talk given at Moriond, 2012.

[67] Tevatron New Physics Higgs Working Group, CDF Collaboration, and D0 Collaboration, "Updated combination of CDF and D0 searches for standard model Higgs boson production with up to $10.0 \mathrm{fb}^{-1}$ of data," http://arxiv.org/abs/1207.0449.

[68] P. H. Frampton, P. Q. Hung, and M. Sher, "Quarks and leptons beyond the third generation," Physics Reports, vol. 330, no. 5-6, pp. 263-348, 2000.

[69] B. Holdom, W. S. Hou, T. Hurth, M. L. Mangano, S. Sultansoy, and G. Unel, "Four statements about the fourth generation," PMC Physics A, vol. 3, article 4, 2009.

[70] D. B. Cline and A. Soni, Eds., The Fourth Family of Quarks and Leptons: First International Symposium, Volume 518, New York Academy of Sciences, New York, NY, USA, 1987.

[71] D. B. Cline and A. Soni, Eds., Annals of the New York Academy of Sciences, Volume 518, New York Academy of Sciences, New York, NY, USA, 1987.

[72] A. Soni, Conference Proceedings C, 871008, 345, 1987.

[73] G. W. S. Hou and A. Soni, in Proceedings of the International Symposium on the Fourth Family of Quarks and Leptons, pp. 255-267, Santa Monica, Calif, USA, 1987.

[74] W. S. Hou and A. Soni, "Does Bd and Bs mixing require the existence of a fourth generation?" Physics Letters B, vol. 196, no. 1, pp. 92-96, 1987.

[75] W. S. Hou, A. Soni, and H. Steger, "Charmless decays of bottom mesons and a fourth generation," Physical Review Letters, vol. 59, no. 14, pp. 1521-1524, 1987.

[76] W. S. Hou, A. Soni, and H. Steger, "Effects of a fourth family on $\mathrm{b} \rightarrow \mathrm{s} \gamma$ and a useful parametrization of quark mixing for rare $\mathrm{B}$ decays," Physics Letters B, vol. 192, no. 3-4, pp. 441-446, 1987.

[77] W. S. Hou, R. S. Willey, and A. Soni, "Implications of a heavy top quark and a fourth generation on the decays $\mathrm{B} \rightarrow \mathrm{Kl}^{+} 1^{-}, \mathrm{K} v^{-}$," Physical Review Letters, vol. 58, no. 16, pp. 1608-1611, 1987.

[78] W. S. Hou, R. S. Willey, and A. Soni, "Implications of a heavy top quark and a fourth generation on the decays $\mathrm{B} \rightarrow \mathrm{Kl}^{+} \mathrm{l}^{-}, \mathrm{Kvv}^{-}$," Physical Review Letters, vol. 60, no. 22, article 2337, 1988.

[79] J. L. Hewett, "Radiative B decays with four generations," Physics Letters B, vol. 193, no. 2-3, pp. 327-330, 1987.

[80] G. Eilam, J. L. Hewett, and T. G. Rizzo, "Four generations and the decay $\mathrm{K} \rightarrow \pi v \bar{v}$," Physics Letters B, vol. 193, no. 4, pp. 533$535,1987$. 
[81] J. L. Hewett and T. G. Rizzo, " $B_{a}^{0}-\bar{B}_{a}^{0}$ mixing in the four generation standard model," Modern Physics Letters A, vol. 3, no. 10 , article $975,1988$.

[82] R. D. Peccei, UCLA/89/TEP/ 34.

[83] J. Beringer, J. F. Arguin, R. M. Barnett et al., "Review of particle physics," Physical Review D, vol. 86, no. 1, Article ID 010001, 1528 pages, 2012.

[84] Y. Fukuda, T. Hayakawa, E. Ichihara et al., "Measurements of the solar neutrino flux from super-Kamiokande's first 300 days," Physical Review Letters, vol. 81, no. 6, pp. 1158-1162, 1998.

[85] Y. Fukuda, T. Hayakawa, E. Ichihara et al., "Erratum: measurements of the solar neutrino flux from super-Kamiokande's first 300 days," Physical Review Letters, vol. 81, p. 4279, 1998, http://arxiv.org/abs/hep-ex/9805021.

[86] M. E. Peskin and T. Takeuchi, "New constraint on a strongly interacting Higgs sector," Physical Review Letters, vol. 65, no. 8, pp. 964-967, 1990.

[87] M. E. Peskin and T. Takeuchi, "Estimation of oblique electroweak corrections," Physical Review D, vol. 46, no. 1, pp. 381409, 1992.

[88] G. D. Kribs, T. Plehn, M. Spannowsky, and T. M. P. Tait, "Four generations and Higgs physics," Physical Review D, vol. 76, no. 7, Article ID 075016, 11 pages, 2007.

[89] J. Erler and P. Langacker, "Precision constraints on extra fermion generations," Physical Review Letters, vol. 105, no. 3, Article ID 031801, 4 pages, 2010.

[90] M. Maltoni, V. A. Novikov, L. B. Okun, A. N. Rozanov, and M. I. Vysotsky, "Extra quark-lepton generations and precision measurements," Physics Letters B, vol. 476, no. 1-2, pp. 107-115, 2000.

[91] V. A. Novikov, L. B. Okun, A. N. Rozanov, and M. I. Vysotsky, "Extra generations and discrepancies of electroweak precision data," Physics Letters B, vol. 529, no. 1-2, pp. 111-116, 2002.

[92] V. A. Novikov, L. B. Okun, A. N. Rozanov, and M. I. Vysotsky, "Mass of the higgs versus fourth generation masses," JETP Letters, vol. 76, no. 3, pp. 127-130, 2002, Pis'ma v Zhurnal Eksperimental'noi i Teoreticheskoi Fiziki, vol. 76, p. 158, 2002.

[93] H. J. He, N. Polonsky, and S. F. Su, "Extra families, Higgs spectrum, and oblique corrections," Physical Review D, vol. 64, no. 5, Article ID 053004, 11 pages, 2001.

[94] J. Alwall, R. Frederix, J. M. Gérard et al., "Is Vtb $\simeq 1$ ?" European Physical Journal C, vol. 49, no. 3, pp. 791-801, 2007.

[95] O. Eberhardt, A. Lenz, and J. Rohrwild, "Less space for a new family of fermions," Physical Review D, vol. 82, no. 9, Article ID 095006, 20 pages, 2010.

[96] C. Promberger, The fourth generation: a comprehensive analysis [Ph.D. thesis], TU Munich, 2011, http://inspirehep.net/ record/1122720/files/1078088.pdf.

[97] M. S. Chanowitz, "Bounding CKM mixing with a fourth family," Physical Review D, vol. 79, no. 11, Article ID 113008, 8 pages, 2009.

[98] M. S. Chanowitz, "Higgs mass constraints on a fourth family: upper and lower limits on CKM mixing," Physical Review D, vol. 82, no. 3, Article ID 035018, 9 pages, 2010.

[99] P. González, J. Rohrwild, and M. Wiebusch, "Electroweak precision observables in a fourth generation model with general flavour structure," European Physical Journal C, vol. 72, no. 5, article 2007, 2012.

[100] O. Eberhardt, G. Herbert, H. Lacker et al., "Joint analysis of Higgs boson decays and electroweak precision observables in the standard model with a sequential fourth generation," Physical Review D, vol. 86, no. 1, Article ID 013011, 7 pages, 2012.

[101] O. Çobanoĝlu, E. Özcan, S. Sultansoy, and G. Ünel, “OPUCEM: a library with error checking mechanism for computing oblique parameters," Computer Physics Communications, vol. 182, no. 8, pp. 1732-1743, 2011.

[102] E. Aşilar, E. Çavlan, O. Doğangün et al., "Oblique parameters and extra generations via OPUCEM," European Physical Journal C, vol. 72, no. 4, article 1966, 2012.

[103] J. Charles, A. Höcker, H. Lacker et al., "CP violation and the CKM matrix: assessing the impact of the asymmetric B factories," European Physical Journal C, vol. 41, no. 1, pp. 1-131, 2005.

[104] M. Ciuchini, V. Lubicz, G. D’Agostini et al., “2000 CKM-triangle analysis. A critical review with updated experimental inputs and theoretical parameters," Journal of High Energy Physics, vol. 2001, no. 7, article 13, 2001.

[105] E. Lunghi and A. Soni, "Possible evidence for the breakdown of the CKM-paradigm of CP-violation," Physics Letters B, vol. 697, no. 4, pp. 323-328, 2011.

[106] A. Lenz, U. Nierste, J. Charles et al., "Anatomy of new physics in $B-\bar{B}$ mixing," Physical Review D, vol. 83 , no. 3 , Article ID 036004, 37 pages, 2011.

[107] V. M. Abazov, B. Abbott, M. Abolins et al., "Evidence for an anomalous like-sign dimuon charge asymmetry," Physical Review D, vol. 82, no. 3, Article ID 032001, 30 pages, 2010.

[108] A. Soni, A. K. Alok, A. Giri, R. Mohanta, and S. Nandi, "The fourth family: a simple explanation for the observed pattern of anomalies in $B-C P$ asymmetries," Physics Letters $B$, vol. 683, no. 4-5, pp. 302-305, 2010.

[109] M. Bobrowski, A. Lenz, J. Riedl, and J. Rohrwild, "How much space is left for a new family of fermions?" Physical Review D, vol. 79, no. 11, Article ID 113006, 15 pages, 2009.

[110] A. Soni, A. K. Alok, A. Giri, R. Mohanta, and S. Nandi, "Standard model with four generations: selected implications for rare $B$ and $K$ decays," Physical Review D, vol. 82, no. 3, Article ID 033009, 23 pages, 2010.

[111] A. J. Buras, B. Duling, T. Feldmann, T. Heidsieck, C. Promberger, and S. Recksiegel, "Patterns of flavour violation in the presence of a fourth generation of quarks and leptons," Journal of High Energy Physics, vol. 2010, no. 9, article 106, 2010.

[112] M. Bobrowski, A. Lenz, J. Riedl, and J. Rohrwild, "How large can the SM contribution to CP violation in $D_{0}-\overline{D_{0}}$ mixing be?" Journal of High Energy Physics, vol. 2010, no. 3, article 9, 2010.

[113] W. S. Hou and C. Y. Ma, "Flavor and CP violation with fourth generations revisited," Physical Review D, vol. 82, no. 3, Article ID 036002, 9 pages, 2010.

[114] A. J. Buras, B. Duling, T. Feldmann, T. Heidsieck, C. Promberger, and S. Recksiegel, "The impact of a 4th generation on mixing and CP violation in the charm system," Journal of High Energy Physics, vol. 2010, no. 7, article 94, 2010.

[115] D. Choudhury and D. K. Ghosh, "A fourth generation, anomalous like-sign dimuon charge asymmetry and the LHC," Journal of High Energy Physics, vol. 2011, no. 2, article 33, 2011.

[116] A. K. Alok, A. Dighe, and D. London, "Constraints on the fourgeneration quark mixing matrix from a fit to flavor-physics data," Physical Review D, vol. 83, no. 7, Article ID 073008, 12 pages, 2011.

[117] S. Nandi and A. Soni, "Constraining the mixing matrix for the standard model with four generations: Time-dependent and semileptonic CP asymmetries in $B_{d}^{0}, B_{s}$, and $D^{0}$," Physical Review D, vol. 83, no. 11, Article ID 114510, 13 pages, 2011. 
[118] R. Mohanta and A. K. Giri, "Study of some rare decays of $B_{s}$ meson in the fourth generation model," Physical Review D, vol. 85, no. 1, Article ID 014008, 11 pages, 2012.

[119] A. N. Rozanov and M. I. Vysotsky, "( $\triangle$ A_CP)_LHCb and the fourth generation," JETP Letters. http://arxiv.org/abs/1111.6949.

[120] A. Lenz, "Theoretical status of the CKM matrix," http://arxiv .org/abs/1108.1218.

[121] A. Lenz, U. Nierste, J. Charles et al., "Constraints on new physics in $B-\bar{B}$ mixing in the light of recent LHCb data," Physical Review $D$, vol. 86, no. 3, Article ID 033008, 5 pages, 2012.

[122] A. Lenz, "Theoretical update of $B$-mixing and lifetimes," http://arxiv.org/abs/1205.1444.

[123] http://belle.kek.jp/.

[124] G. Aad, B. Abbott, J. Abdallah et al., "Search for pair production of a heavy up-type quark decaying to a W boson and a b quark in the lepton+jets channel with the ATLAS detector," Physical Review Letters, vol. 108, no. 26, Article ID 261802, 18 pages, 2012.

[125] G. Aad, B. Abbott, J. Abdallah et al., "Search for pair-produced heavy quarks decaying to Wq in the two-lepton channel at $\sqrt{(s)}=7 \mathrm{TeV}$ with the ATLAS detector," Physical Review D, vol. 86, no. 1, Article ID 012007, 24 pages, 2012.

[126] G. Aad, B. Abbott, J. Abdallah et al., "Search for down-type fourth generation quarks with the ATLAS detector in events with one Lepton and hadronically decaying W bosons," Physical Review Letters, vol. 109, no. 3, Article ID 032001, 19 pages, 2012.

[127] S. Chatrchyan CMS Collaboration, "Search for heavy, top-like quark pair production in the dilepton final state in pp collisions at $\sqrt{s}=7$ TeV," Physics Letters B, vol. 716, no. 1, pp. 103-121, 2012.

[128] S. Chatrchyan, V. Khachatryan, A. M. Sirunyan et al., "Search for heavy bottom-like quarks in $4.9 \mathrm{fb}^{-1}$ of pp collisions at $\sqrt{s}=$ 7 TeV," Journal of High Energy Physics, vol. 2012, no. 5, article 123, 2012.

[129] G. Aad, B. Abbott, J. Abdallah et al., "Search for pair production of a new b' quark that decays into a $\mathrm{Z}$ boson and a bottom quark with the ATLAS detector," Physical Review Letters, vol. 109, no. 7, Article ID 071801, 19 pages, 2012.

[130] C. J. Flacco, D. Whiteson, and M. Kelly, "Fourth-generation quark mass limits in CKM-element space," Physical Review D, vol. 83, no. 11, Article ID 114048, 8 pages, 2011.

[131] J. F. Gunion, D. W. McKay, and H. Pois, "Minimal four-family supergravity model," Physical Review D, vol. 53, no. 3, pp. 16161647, 1996.

[132] J. Ellis, M. K. Gaillard, and D. V. Nanopoulos, "A phenomenological profile of the Higgs boson," Nuclear Physics B, vol. 106, pp. 292-340, 1976.

[133] B. L. Ioffe and V. A. Khoze, Soviet Journal of Particles and Nuclei, vol. 9, p. 50, 1978, B. L. Ioffe and V. A. Khoze, Fizika Elementarnykh Chastits i Atomnoya Yadra, vol. 9, p. 118, 1978.

[134] M. A. Shifman, A. I. Vainshtein, M. B. Voloshin, and V. I. Zakharov, Soviet Journal of Nuclear Physics, vol. 30, p. 711, 1979, M. A. Shifman, A. I. Vainshtein, M. B. Voloshin and V. I. Zakharov, Yadernaya Fizika, vol. 30, 1979.

[135] L. H. Chan and T. Hagiwara, "Can we measure the number of quark flavors without building an ultrahigh-energy accelerator?” Physical Review D, vol. 20, no. 7, pp. 1698-1703, 1979.

[136] J. F. Gunion, "Ruling out a 4th generation using limits on hadron collider Higgs signals," http://arxiv.org/abs/1105.3965.

[137] X. Ruan and Z. Zhang, "Impact on the Higgs production cross section and decay branching fractions of heavy quarks and Leptons in a fourth generation model," http:// arxiv.org/abs/1105.1634.
[138] G. Guo, B. Ren, and X. G. He, "LHC evidence of a $126 \mathrm{GeV}$ Higgs boson from $\$ \mathrm{H}$ to $\gamma \gamma \$$ with three and four generations," http://arxiv.org/abs/1112.3188.

[139] M. E. Peskin and arXiv:1, "Summary of Lepton photon 2011," http://arxiv.org/abs/1110.3805.

[140] V. A. Khoze, "Comment on an invisible Higgs boson and $50 \mathrm{GeV}$ neutrino," http://arxiv.org/abs/hep-ph/0105069.

[141] K. Belotsky, D. Fargion, M. Khlopov, R. Konoplich, and K. Shibaev, "Invisible Higgs boson decay into massive neutrinos of fourth generation," Physical Review D, vol. 68, no. 5, Article ID 054027, 4 pages, 2003.

[142] S. S. Bulanov, V. A. Novikov, L. B. Okun, A. N. Rozanov, and M. I. Vysotsky, "Z lineshape versus fourth-generation masses," Physics of Atomic Nuclei, vol. 66, no. 12, pp. 2169-2177, 2003, Yadernaya Fizika, vol. 66, no. 12, pp. 2219-2227, 2003.

[143] A. N. Rozanov and M. I. Vysotsky, "Tevatron constraints on the Higgs boson mass in the fourth-generation fermion models revisited," Physics Letters B, vol. 700, no. 5, pp. 313-315, 2011.

[144] S. A. Çetin, T. Çuhadar-Dönszelmann, M. Şahin, S. Sultansoy, and G. Ünel, "Impact of the relatively light fourth family neutrino on the Higgs boson search," Physics Letters B, vol. 710, no. 2, pp. 328-331, 2012.

[145] C. Englert, J. Jaeckel, E. Re, and M. Spannowsky, "Evasive Higgs boson maneuvers at the LHC," Physical Review D, vol. 85, no. 3, Article ID 035008, 10 pages, 2012.

[146] T. Uhadar-Dönszelmann, M. Karagöz Nel, V. E. Zcan, S. Sultansoy, and G. Önel, "Fourth family neutrinos and the Higgs boson at the LHC," Journal of High Energy Physics, vol. 2008, no. 10 , article 74, 2008.

[147] L. M. Carpenter and A. Rajaraman, "Revisiting constraints on fourth generation neutrino masses," Physical Review D, vol. 82, no. 11, Article ID 114019, 5 pages, 2010.

[148] W. Y. Keung and P. Schwaller, "Long lived fourth generation and the Higgs," Journal of High Energy Physics, vol. 2011, no. 6, article 54, 2011.

[149] L. M. Carpenter, "Higgs search constraints on fourth generation scenarios with general Lepton sectors," http://arxiv .org/abs/1110.4895.

[150] G. Passarino, C. Sturm, and S. Uccirati, "Complete electroweak corrections to Higgs production in a Standard Model with four generations at the LHC," Physics Letters B, vol. 706, no. 2-3, pp. 195-199, 2011.

[151] A. Djouadi, J. Kalinowski, and M. Spira, "HDECAY: a program for Higgs boson decays in the standard model and its supersymmetric extension," Computer Physics Communications, vol. 108, no. 1, pp. 56-74, 1998.

[152] Programme HDecay avaialable at: http://people.web.psi.ch/ spira/hdecay/.

[153] E. Kuflik, Y. Nir, and T. Volansky, "Implications of Higgs searches on the four generation standard model," http:// arxiv.org/abs/1204.1975.

[154] M. Buchkremer, J.-M. Gérard, and F. Maltoni, "Closing in on a perturbative fourth generation," Journal of High Energy Physics, vol. 2012, no. 6, article 135, 2012.

[155] The ATLAS collaboration, "Update of the combination of Higgs boson searches in pp collisions at $\sqrt{(s)}=7 \mathrm{TeV}$ with the ATLAS experiment at the LHC," in Proceedings of the 25th International Symposium on Lepton Photon Interactions at High Energies, Mumbai, India, August 2011, ATLAS-CONF-2011-135.

[156] “Combination of SM, SM4, FP Higgs boson searches," CMS PAS HIG-12-008. 
[157] M. Wiebusch, "Numerical Computation of p-values with myFitter," http://arxiv.org/abs/1207.1446.

[158] O. Eberhardt, G. Herbert, H. Lacker et al., "Impact of a Higgs boson at a mass of $126 \mathrm{GeV}$ on the standard model with three and four fermion generations," Physical Review Letters. http://arxiv.org/abs/1209.1101.

[159] P. Gerhold, K. Jansen, and J. Kallarackal, "Higgs boson mass bounds in the presence of a very heavy fourth quark generation," Journal of High Energy Physics, vol. 2011, no. 1, article 143, 2011.

[160] M. A. Luty, "Dynamical electroweak symmetry breaking with two composite Higgs doublets," Physical Review D, vol. 41, no. 9, pp. 2893-2902, 1990.

[161] S. Bar-Shalom, S. Nandi, and A. Soni, "Two Higgs doublets with fourth-generation fermions: models for TeV-scale compositeness," Physical Review D, vol. 84, no. 5, Article ID 053009, 24 pages, 2011.

[162] X. G. He and G. Valencia, "An extended scalar sector to address the tension between a fourth generation and Higgs searches at the LHC," Physics Letters B, vol. 707, no. 3-4, pp. 381-384, 2012.

[163] S. Bar-Shalom, S. Nandi, and A. Soni, "Muon g-2 and lepton flavor violation in a two Higgs doublets model for the fourth generation," Physics Letters B, vol. 709, no. 3, pp. 207-217, 2012.

[164] N. Chen and H. J. He, "LHC signatures of two-Higgs-doublets with fourth family," Journal of High Energy Physics, vol. 2012, no. 4, article 62, 2012.

[165] M. Geller, S. Bar-Shalom, and G. Eilam, "The need for new search strategies for fourth generation quarks at the LHC," Physics Letters B, vol. 715, no. 1-3, pp. 121-128, 2012.

[166] L. Bellantoni, J. Erler, J. J. Heckman, and E. Ramirez-Homs, "Masses of a fourth generation with two Higgs doublets," Physical Review D, vol. 86, no. 3, Article ID 034022, 9 pages, 2012.

[167] A. Lenz, H. Päs, and D. Schalla, "Fourth generation Majorana neutrinos," Physical Review D, vol. 85, no. 7, Article ID 075025, 8 pages, 2012. 

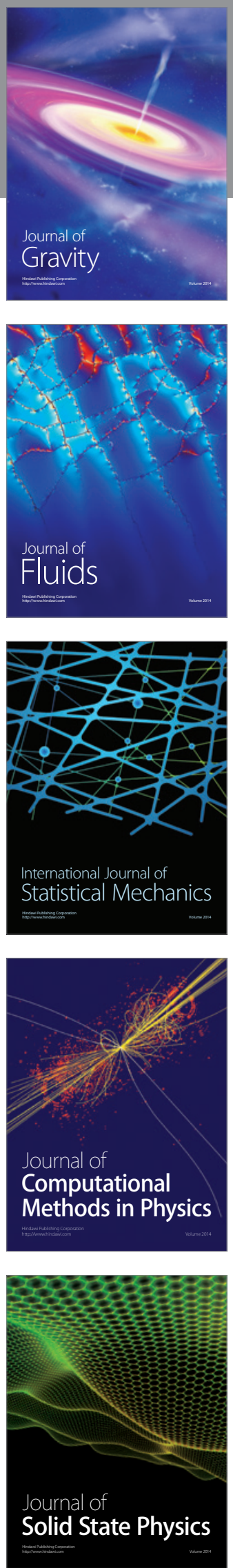

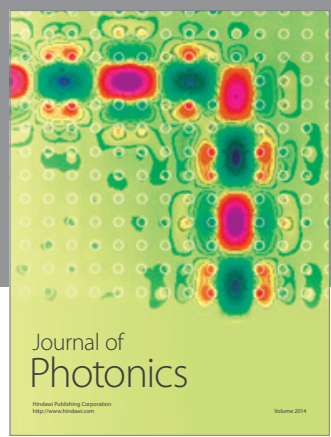

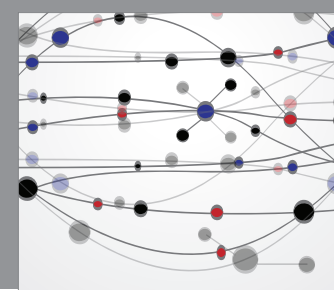

The Scientific World Journal

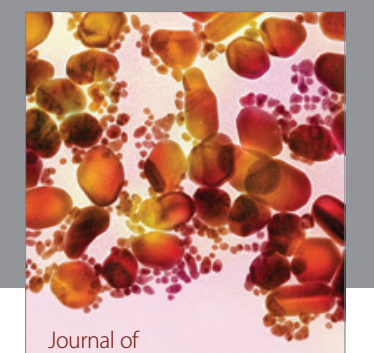

Soft Matter
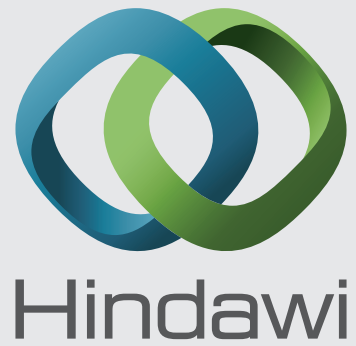

Submit your manuscripts at

http://www.hindawi.com
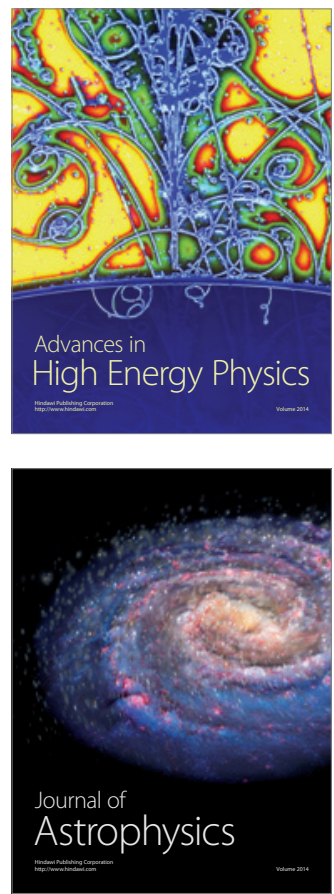
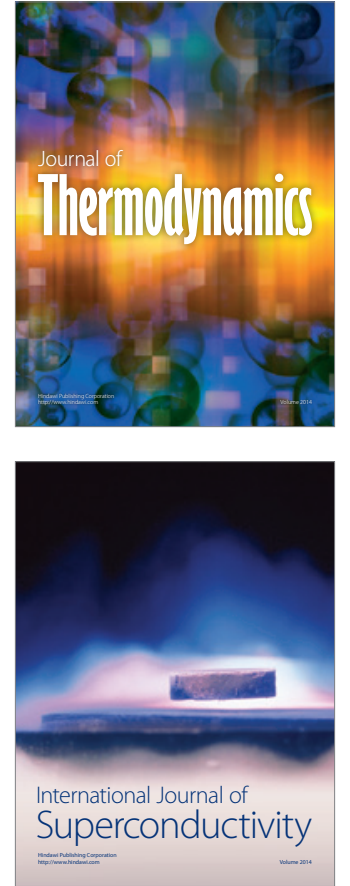
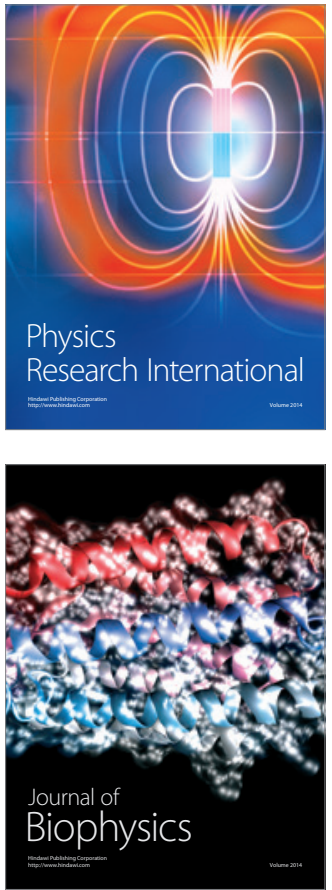
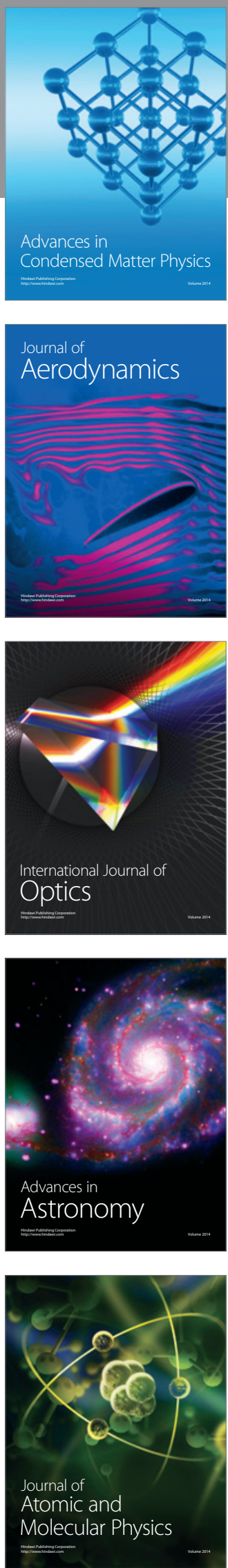